\title{
Objective evaluation of the alleviating effects of Goshajinkigan on peripheral neuropathy induced by paclitaxel/carboplatin therapy: A multicenter collaborative study
}

\author{
HIROI KAKU $^{1}$, SEISUKE KUMAGAI ${ }^{1}$, HIROKI ONOUE ${ }^{1}$, ANNA TAKADA ${ }^{1}$, TADAHIRO SHOJI ${ }^{1}$, \\ FUMIHARU MIURA $^{1}$, AKIRA YOSHIZAKI ${ }^{1}$, SHINYA SATO ${ }^{2}$, JUNZO KIGAWA ${ }^{3}$, TSUTOMU ARAI ${ }^{4}$, \\ SHINPEI TSUNODA ${ }^{4}$, EIICHIRO TOMINAGA ${ }^{5}$, DAISUKE AOKI ${ }^{5}$ and TORU SUGIYAMA ${ }^{1}$ \\ ${ }^{1}$ Department of Obstetrics and Gynecology, Iwate Medical University, Iwate 020-8505; \\ ${ }^{2}$ Department of Obstetrics and Gynecology; ${ }^{3}$ Cancer Center, Tottori University Hospital, Tottori 683-8504; \\ ${ }^{4}$ Department of Obstetrics and Gynecology, Kitasato University Hospital, Kanagawa 252-0375; \\ ${ }^{5}$ Department of Obstetrics and Gynecology, Keio University Hospital, Tokyo 160-8582, Japan
}

Received July 25, 2011; Accepted September 9, 2011

DOI: $10.3892 / \mathrm{etm} .2011 .375$

\begin{abstract}
Paclitaxel/carboplatin chemotherapy for cancer (TC therapy) exhibits neurotoxicity and causes peripheral neuropathy at a high frequency, which is difficult to cope with. In this study, we investigated the efficacy of Goshajinkigan, a traditional Japanese herbal medicine, for TC therapy-induced peripheral neuropathy. The subjects included in our study were patients with ovarian or endometrial cancer who underwent TC therapy and developed peripheral neuropathy. The patients were randomly divided into Group A, comprising of 14 patients (vitamin B12 treatment), and Group B, comprising of 15 patients (vitamin B12 + Goshajinkigan treatment). The observation period was 6 weeks following treatment initiation, and the evaluation items were as follows: i) the current perception threshold (CPT value) of the peripheral nerve, ii) visual analogue scale for numbness, iii) National Cancer Institute Common Terminology Criteria for Adverse Events v3.0 grade of neurotoxicity, and iv) a questionnaire on the subjective symptoms of peripheral neuropathy (functional assessment of cancer therapy-taxane). These were compared between the groups and no significant differences were noted in any item. However, CTCAE grade 3 neurotoxicity developed in 2 patients (14.3\%) after 6 weeks of administration in Group A, whereas no neurotoxicity was observed in Group B. When the change in the frequency of abnormal CPT ratio at 6 weeks of administration from that before treatment was compared between the groups, the frequency of abnormal value was
\end{abstract}

Correspondence to: Dr Toru Sugiyama, Department of Obstetrics and Gynecology, Iwate Medical University, 19-1 Uchimaru, Morioka, Iwate 020-8505, Japan

E-mail: hiroi1216jp@yahoo.co.jp

Key words: gynecological cancer, kampo drugs, Japanese herbal medicine, paclitaxel, Goshajinkigan, neurotoxicity, peripheral neuropathy significantly lower in Group B than in Group A $(\mathrm{p}<0.05)$. This suggests that Goshajinkigan inhibits the progression of peripheral neuropathy.

\section{Introduction}

For chemotherapy against gynecological cancer centering on ovarian cancer, platinum and taxane preparations are widely used. However, neurotoxicity, especially peripheral neuropathy appearing as an adverse reaction to a taxane preparation, paclitaxel, is a problem that remains to be solved. Several patients develop intractable nervous symptoms persisting for months after receiving the paclitaxel treatment, and this is one of the factors that cause deterioration of patient quality of life (QOL). Recently, it was reported that Goshajinkigan, a traditional Japanese herbal medicine, is useful for coping with chemotherapy-induced peripheral neuropathy (1).

However, in general, it is difficult to objectively evaluate the severity of peripheral neuropathy, and very few reports have referred to the objective electrophysiological evaluation of neuropathy. We tried to undertake objective evaluations of neuropathy, including the determination of current perception thresholds (CPT) in gynecological patients who underwent chemotherapy including paclitaxel, and developed peripheral neuropathy. Such patients were randomly assigned to two groups receiving and not receiving the Goshajinkigan treatment, and the efficacy of Goshajinkigan in alleviating peripheral neuropathy was investigated.

\section{Patients and methods}

Patients. This study was conducted under the approval of the Institutional Review Board (IRB) of each study center. The subjects were patients with ovarian or endometrial cancer who met all of the following inclusion criteria: i) histological diagnosis of ovarian or endometrial cancer, ii) having at least one cycle of paclitaxel and carboplatin combination therapy (TC therapy) conducted as the first chemotherapy and a National 
Table I. Evaluation items.

\begin{tabular}{lc}
\hline Evaluation item & Range \\
\hline $\begin{array}{l}\text { Evaluation of current perception } \\
\text { threshold (CPT) } \\
\text { (evaluation of forefinger) }\end{array}$ & $0-999(100=1 \mathrm{mAmp})$ \\
$\begin{array}{l}\text { Grade of numbness on visual } \\
\text { analogue scale (VAS) }\end{array}$ & $0-10$ \\
$\begin{array}{l}\text { Motor and sensory neuropathy grade } \\
\text { (NCI-CTCAE v3.0) }\end{array}$ & Grade 0 \\
& (no symptom) - \\
$\begin{array}{l}\text { Subjective neuropathy symptom } 5 \\
\text { questionnaire examination } \\
\text { (FACT-Taxane) }\end{array}$ & $0-64$ \\
\hline
\end{tabular}

The items were evaluated before the study treatment and after 3 and 6 weeks of the study treatment, and the changes were compared between the Goshajinkigan treatment group and the Goshajinkigan non-treatment group.

Cancer Institute Common Terminology Criteria for Adverse Events v3.0 (NCI-CTCAE) peripheral neuropathy grade of $\geq 1$, iii) age of $\geq 20$ and $\leq 70$ years, and iv) having provided written consent to participate in this study. The exclusion criteria were as follows: i) previous use of Goshajinkigan or vitamin B12 within the past 4 weeks, ii) any schedule of using another anticancer agent during the study period, and iii) presence of severe peripheral neuropathy at the initiation of this study.

Methods. This study was conducted as a parallel group randomized controlled trial by central registration, and the registered patients were randomly assigned to the Goshajinkigan nontreatment group (control group, Group A) and the Goshajinkigan treatment group (Group B). In Group B, Tsumura Goshajinkigan Extract Granules ${ }^{\circledR} 7.5 \mathrm{~g} /$ day (t.i.d.) and vitamin B12 (Methycobal ${ }^{\circledR}$ ) at $1,500 \mu \mathrm{g} /$ day (t.i.d.) were administered, and in Group A, only vitamin B12 was administered. The registered patients received a maximum of 6 cycles of TC therapy (paclitaxel 175-180 mg/m², i.v. on Day 1; carboplatin AUC 5-6 , i.v. on Day 1; each cycle, 21 days).

Evaluation items. Patients were observed for 6 weeks, and they underwent CPT determination of the bilateral forefingers, visual analogue scale (VAS) determination for numbness, grade classification of motor and sensory neuropathy according to CTCAE and examination with the subjective neuropathy symptom questionnaire using modified functional assessment of cancer therapy-taxane (FACT-Taxane) (2) before the study treatment, after 3 weeks, and after 6 weeks of the study treatment to compare the changes in neuropathy symptoms between the two groups (Tables I and II).

CPT test. The CPT examination has been reported to be useful for the detection, screening, diagnosis and management of diseases of peripheral neuropathy (3-5). The principle and method of CPT determination are shown below. The nerve diameter differs depending on the nerve type, and each nerve
Table II. Subjective neuropathy symptom questionnaire using modified FACT-Taxane.

Upper limb symptoms

Hand numbness or tingling pain

Other uncomfortable hand sensation

Bilateral hand swelling

Sore fingertips

Trouble in buttoning

Difficulty in feeling the shape of a small object

grasped in the hand

Lower limb symptoms

Foot numbness or tingling pain

Other uncomfortable foot sensation

Bilateral foot swelling

Difficulty in walking

Other symptoms

Joint pain or muscle convulsion

Whole body swelling

Feeling of whole body weakness

Worsened hearing acuity

Noise in the ear

Very concerned about unusual appearance of hands and nails

Self-entry questionnaire about upper/lower limb symptoms and other symptoms; each response was scored depending on severity ( 0 points, not applicable at all; 1 point, slightly applicable; 2 points, somewhat applicable; 3 points, considerably applicable; 4 points, very applicable).

Table III. Normative data for median nerve (100 CPT $=1 \mathrm{mAmp})$.

\begin{tabular}{lrrrr}
\hline & Min & Max & Mean & SD \\
\hline Ranges $(\mathrm{Hz})$ & & & & \\
2,000 & 120 & 398 & 226 & 80 \\
250 & 22 & 180 & 81 & 42 \\
5 & 16 & 101 & 46 & 27 \\
Within-site ratio & & & & \\
$2 \mathrm{kHz} / 5 \mathrm{~Hz}$ & 2.03 & 14.7 & 6.2 & 4.2 \\
$2 \mathrm{kHz} / 250 \mathrm{~Hz}$ & 1.53 & 5.80 & 3.2 & 2.1 \\
$250 \mathrm{~Hz} / 5 \mathrm{~Hz}$ & 0.83 & 4.38 & 2.0 & 1.1 \\
\end{tabular}

Sources: Neuval ${ }^{\circledR}$ Database II - Normative Data, Neurotron, Inc., Baltimore, MD, USA, 2001. These normative data were obtained primarily from the following institutions: Johns Hopkins Medical Institution, University of Maryland School of Pharmacology, Creighton University School of Medicine, New York Medical College, New York University Medical Center, Palmer College of Chiropractic and the University of New Mexico School of Medicine.

has a specific frequency suitable for depolarization depending on its diameter. It is therefore possible to undertake selective quantitative evaluation of both large fibers and small fibers by stimulating at different frequencies. The sine-wave current is gradually increased from a low level (0-9.99 mA) at three different frequencies of 5,250 and $2,000 \mathrm{~Hz}$ at the region of measurement. The minimum current perceived is the CPT 
Table IV. Patient background.

\begin{tabular}{|c|c|c|c|}
\hline & $\begin{array}{l}\text { Group A (not administered } \\
\text { Goshajinkigan; } n=14 \text { ) }\end{array}$ & $\begin{array}{l}\text { Group B (administered } \\
\text { Goshajinkigan; } n=15 \text { ) }\end{array}$ & $\begin{array}{c}\text { Wilcoxon's rank } \\
\text { sum test }\end{array}$ \\
\hline Age (years) & 59.7 & 55.6 & \\
\hline \multicolumn{4}{|l|}{ Performance status } \\
\hline 0 & 9 & 9 & \\
\hline 1 & 5 & 4 & \\
\hline 2 & 0 & 2 & \\
\hline \multicolumn{4}{|l|}{ Disease } \\
\hline Ovarian cancer & 12 & 12 & \\
\hline Endometrial cancer & 2 & 2 & \\
\hline Multiple cancer & 0 & 1 & \\
\hline Vas score & $3.1 \pm 2.2$ & $2.5 \pm 1.6$ & $\mathrm{p}=0.827$ \\
\hline \multicolumn{4}{|c|}{ CTCAE neuropathy grade } \\
\hline Motor & $0.6 \pm 0.7$ & $0.5 \pm 0.7$ & $\mathrm{p}=0.760$ \\
\hline Sensory & $1.3 \pm 0.5$ & $1.1 \pm 0.4$ & $\mathrm{p}=0.404$ \\
\hline FACT-Taxane & $8.5 \pm 5.5$ & $8.3 \pm 8.1$ & $\mathrm{p}=0.896$ \\
\hline \multicolumn{4}{|l|}{ CPT range $(5 \mathrm{~Hz})$} \\
\hline Right & $113 \pm 68.3$ & $98 \pm 46.6$ & $\mathrm{p}=0.535$ \\
\hline Left & $120 \pm 62.6$ & $121 \pm 117.9$ & $\mathrm{p}=0.451$ \\
\hline \multicolumn{4}{|l|}{ CPT range $(250 \mathrm{~Hz})$} \\
\hline Right & $156 \pm 73.3$ & $168 \pm 92.1$ & $\mathrm{p}=0.947$ \\
\hline Left & $167 \pm 63.4$ & $163 \pm 105.1$ & $\mathrm{p}=0.385$ \\
\hline \multicolumn{4}{|l|}{ CPT range $(2 \mathrm{kHz})$} \\
\hline Right & $346 \pm 140.8$ & $375 \pm 127.2$ & $\mathrm{p}=0.537$ \\
\hline Left & $366 \pm 138.8$ & $341 \pm 133.6$ & $\mathrm{p}=0.354$ \\
\hline
\end{tabular}

Patient backgrounds at the time of registration in this study. The patients were randomly assigned to the Goshajinkigan non-treatment group (Group A) or the Goshajinkigan treatment group (Group B). There were no significant inter-group differences in patient backgrounds.

of each subject. The CPT at 2,000 Hz corresponds to a large myelinated fiber $(\mathrm{A} \beta)$, and the $\mathrm{CPT}$ at $250 \mathrm{~Hz}$ corresponds to a small myelinated fiber (Aס), while the CPT at $5 \mathrm{~Hz}$ corresponds to an unmyelinated nerve (C) (6).

CPT was measured using the Neurometer NervScan NS3000 ${ }^{\circledR}$. CPT range and within-site CPT ratio analyses of the bilateral second fingers controlled by the median nerves were performed.

The CPT Range Analysis compares raw CPT measures to the normative ranges. $\mathrm{CPT}$ values below the minimum $\mathrm{CPT}$ normative range qualify as hyperesthesia and indicate that the nerve fibers are suffering from inflammation or are under regeneration, and values above the maximum CPT normative range indicate hypoesthesia associated with loss of function or neuropathy.

The within-site CPT ratio analysis is an analytical method for measureing the ratio within the measurement region $(2,000 \mathrm{~Hz} / 5 \mathrm{~Hz} ; 2,000 \mathrm{~Hz} / 250 \mathrm{~Hz} ; 250 \mathrm{~Hz} / 5 \mathrm{~Hz})$. Ratios outside the healthy ranges indicate very mild sensory abnormalities. The normative data are displayed in Table III.

Statistical analysis. Statistical analysis was performed using statistical analysis software, SAS release 9.13 (SAS
Institute Japan). The VAS value, FACT-Taxane score, CTCAE neuropathy grade and therapeutic effect on CPT (2,000, 250 or $5 \mathrm{~Hz}$ ) were evaluated at each time-point in each group employing Wilcoxon's signed rank test. Wilcoxon's rank sum test was employed for the comparison of the frequencies of the abnormal values of the above evaluation items and the CPT test between Group A and B. Since this was an exploratory study, multiplicity was not considered in any test. A value of $\mathrm{p}<0.05$ was regarded as significant for all data.

\section{Results}

In the period from March 8, 2007, to March 31, 2009, 31 patients were registered at the four study centers (Iwate Medical University Hospital, Tottori University Hospital, Kitasato University Hospital and Keio University Hospital). All the patients met the inclusion criteria, although 2 patients dropped out of this study since they developed deep vein thrombosis in the lower limb during the TC therapy. Therefore, 29 valid patients (Group A, 15 patients; Group B, 14 patients) were included in the analysis. The patient background factors did not differ between the two groups (Table IV). 
Table V. Changes in the outcome.

\begin{tabular}{|c|c|c|c|c|c|c|c|}
\hline & \multicolumn{3}{|c|}{$\begin{array}{l}\text { Group A (not administered } \\
\text { Goshajinkigan; } n=14 \text { ) }\end{array}$} & \multicolumn{3}{|c|}{$\begin{array}{l}\text { Group B (administered } \\
\text { Goshajinkigan; } n=15 \text { ) }\end{array}$} & \multirow[t]{2}{*}{$\begin{array}{l}\text { Wilcoxon's rank } \\
\text { sum test }{ }^{\mathrm{a}}\end{array}$} \\
\hline & 0 weeks & 3 weeks & 6 weeks & 0 weeks & 3 weeks & 6 weeks & \\
\hline VAS & $3.1 \pm 2.2$ & $2.9 \pm 3.3$ & $3.7 \pm 3.4$ & $2.5 \pm 1.6$ & $3.8 \pm 2.7$ & $3.4 \pm 2.7$ & $\mathrm{p}=0.827$ \\
\hline \multicolumn{8}{|l|}{ CTCAE grade } \\
\hline Motor neuropathy & $0.6 \pm 0.7$ & $0.6 \pm 1.0$ & $0.8 \pm 1.1$ & $0.5 \pm 0.7$ & $0.6 \pm 0.7$ & $0.8 \pm 0.9$ & $\mathrm{p}=0.760$ \\
\hline Sensory neuropathy & $1.3 \pm 0.5$ & $1.0 \pm 1.0$ & $1.3 \pm 1.0$ & $1.1 \pm 0.4$ & $1.3 \pm 0.5$ & $1.4 \pm 0.5$ & $\mathrm{p}=0.404$ \\
\hline FACT-Taxane & $8.5 \pm 5.5$ & $6.8 \pm 6.9$ & $8.9 \pm 8.4$ & $8.3 \pm 8.1$ & $9.9 \pm 7.1$ & $8.2 \pm 7.0$ & $\mathrm{p}=0.896$ \\
\hline \multicolumn{8}{|l|}{ CPT range $(5 \mathrm{~Hz})$} \\
\hline Right & $113 \pm 68.3$ & $119 \pm 59.6$ & $137 \pm 171.1$ & $98 \pm 46.6$ & $110 \pm 94.4$ & $95 \pm 41.1$ & $\mathrm{p}=0.793$ \\
\hline Left & $120 \pm 62.6$ & $141 \pm 84.4$ & $154 \pm 147.6$ & $121 \pm 117.9$ & $112 \pm 87.2$ & $119 \pm 66.3$ & $\mathrm{p}=0.948$ \\
\hline \multicolumn{8}{|l|}{$\mathrm{CPT}$ range $(250 \mathrm{~Hz})$} \\
\hline Right & $156 \pm 73.3$ & $141 \pm 67.6$ & $140 \pm 80.7$ & $168 \pm 92.1$ & $151 \pm 122.9$ & $148 \pm 30.6$ & $\mathrm{p}=0.982$ \\
\hline Left & $167 \pm 63.4$ & $164 \pm 76.7$ & $159 \pm 127.0$ & $163 \pm 105.1$ & $169 \pm 93.0$ & $164 \pm 57.8$ & $\mathrm{p}=0.444$ \\
\hline \multicolumn{8}{|l|}{$\mathrm{CPT}$ range $(2,000 \mathrm{~Hz})$} \\
\hline Right & $346 \pm 140.8$ & $360 \pm 63.3$ & $340 \pm 96.6$ & $375 \pm 127.2$ & $347 \pm 103.5$ & $347 \pm 109.7$ & $\mathrm{p}=0.611$ \\
\hline Left & $366 \pm 138.8$ & $393 \pm 147.7$ & $355 \pm 155.5$ & $341 \pm 133.6$ & $334 \pm 90.6$ & $358 \pm 106.3$ & $\mathrm{p}=0.743$ \\
\hline
\end{tabular}

${ }^{a} 0$ vs. 6 weeks. The duration of observation was 6 weeks. There were no significant differences in the VAS score, CTCAE grade, FACT-Taxane or CTP grade between the groups.

Table VI. Changes in CTCAE (sensory neuropathy) grade.

\begin{tabular}{lccc}
\hline CTCAE sensory neuropathy & $\begin{array}{c}\text { Before study } \\
\text { treatment }\end{array}$ & $\begin{array}{c}\text { After } 3 \text { weeks } \\
\text { of study treatment }\end{array}$ & (no.) \\
of study treatment & (no.) & (no.) & (no.) \\
\hline
\end{tabular}

Group A (not administered Goshajinkigan)

$\begin{array}{lrrr}\text { No symptom } & 0 & 5 & 3 \\ \text { Grade } 1 & 10 & 5 & 6 \\ \text { Grade } 2 & 4 & 3 & 3 \\ \text { Grade } 3 & 0 & 1 & 2 \\ \text { Grade } 4 & 0 & 0 & 0 \\ \text { Grade } 5 & 0 & 0 & 0\end{array}$

Group B (administered Goshajinkigan)

$\begin{array}{lrrr}\text { No symptom } & 0 & 0 & 0 \\ \text { Grade } 1 & 13 & 11 & 9 \\ \text { Grade } 2 & 2 & 4 & 6 \\ \text { Grade } 3 & 0 & 0 & 0 \\ \text { Grade } 4 & 0 & 0 & 0 \\ \text { Grade } 5 & 0 & 0 & 0\end{array}$

When limited to grade classification of sensory neuropathy, an event of $\geq$ Grade 3 was noted in 2 patients (14.3\%) of the Goshajinkigan nontreatment group (Group A), but no events were noted in the Goshajinkigan treatment group (Group B).

There were no significant differences between Group A and Group B in terms of changes in VAS score, CTCAE neuropathy grade, FACT-Taxane and CPT ranges in the period from before the study treatment to Week 6 of study treatment (Table V). However, when limited to the grade of sensory neuropathy, symptoms of Grade $\geq 3$ were noted in Group A at Week 3 or later (Week 6, 14.3\%), but not in Group B (Table VI).

The change in the frequency of abnormal CPT range at 6 weeks of administration from that before treatment was compared between the groups employing Wilcoxon's rank 


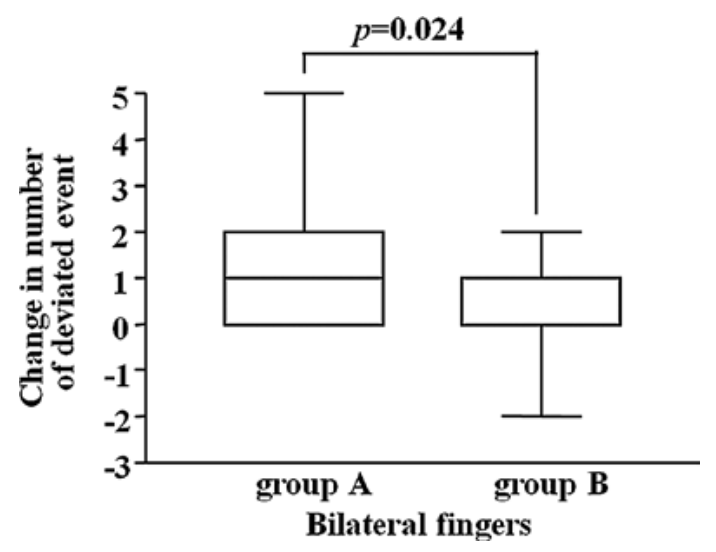

Figure 1. The change in the number of incidences of abnormal CPT ratio at 6 weeks of administration from that before treatment, was analyzed. The incidence of abnormal value was significantly lower in Group B than in Group A (Wilcoxon's rank sum test, 0 vs. 6 weeks).

sum test, but no significant difference was observed. However, regarding the change in the frequency of abnormal CPT ratio, the frequency was significantly lower in Group B than in Group A (Fig. 1).

\section{Discussion}

Paclitaxel, irinotecan hydrochloride and liposomal doxorubicin, among others, have specific non-hematological toxicities, inducing the deterioration of patient QOL. Therefore, it is important to decrease such toxicities. For neurotoxicity in particular, peripheral neuropathy is known as an adverse reaction to paclitaxel (7). Vasey et al (8) reported that the incidence of peripheral neuropathy in TC therapy was as high as $78 \%$ for sensory disorder and $16 \%$ for motor disorder. Recently, it was reported that Goshajinkigan, a traditional Japanese herbal medicine, is effective for chemotherapy-induced neurotoxicity. However, there are few reports on the electrophysiological evaluation of the severity of peripheral neuropathy.

In this study, we evaluated the peripheral neuropathyalleviating effects of Goshajinkigan administered to patients with gynecological malignancy undergoing TC therapy in a parallel group randomized controlled trial. There were no significant differences between the presence and absence of Goshajinkigan treatment after 3 and 6 weeks of the study treatment in VAS evaluation for numbness, subjective neuropathy symptom questionnaire using FACT-Taxane and neuropathy grade according to CTCAE. Since an anticancer drug induces not only neuropathy, but also various complications, such as gastrointestinal symptoms, infections and mental symptoms, it is difficult to avoid biases in the cases of VAS and FACTTaxane. This was considered to be the cause of the failure to obtain significant differences. However, in CTCAE for sensory neuropathy, an event of Grade 3 was noted in 2 patients $(14.3 \%)$ of the Goshajinkigan non-treatment group, while no event of Grade 3 was noted in the Goshajinkigan treatment group. This suggests that the progression of neuropathy can be delayed by the use of Goshajinkigan.
It is generally difficult to evaluate the severity of peripheral neuropathy objectively. The CPT-measuring method has gradually prevailed since it was developed by Katims et al (9) in 1986, showing a high prevalence rate in the United States. It has been reported that this method can be used to make detailed neurological evaluations of diabetic peripheral neuropathy, carpal tunnel syndrome and alcoholic peripheral neuropathy (3-5).

No significant difference was noted in the changes in the CPT range between the groups with and without Goshajinkigan treatment, but the frequency of abnormal CPT ratio was significantly lower in the Goshajinkigan-treatment group. The progression of TC therapy-induced neurotoxicity over the 6-week observation period was not so marked so that it was not reflected in the CPT range, showing no significant difference. By contrast, the CPT ratio showed a significant difference as it may have reflected very mild sensory abnormalities, suggesting that Goshajinkigan inhibited the very early progression of neurotoxicity.

For the prevention and treatment of peripheral neuropathy induced by anticancer agents, various animal experiments and clinical studies were conducted using, in addition to NSAIDs and steroids, antihistaminic drugs, NGF (10,11), IGF (12), GDNF (13), amifostine (14,15), glutathione (16), $\alpha$-lipoic acid (17), gabapentin (18) and carbamazepine (19), among others, but there are no established methods.

Goshajinkigan is a kampo drug prepared by adding Goshitsu (Achyranthes root, anti-allergic effect) and Shazenshi (Plantago seed, diuretic effect and interferon-inducing effect) to Hachimijiogan, which is composed of the following mixed crude drugs: Rehmanma root, Cornus fruit, Dioscorea rhizome, Alisma rhizome, Poria sclerotium, Moutan bark, Cinnamon bark and processed Aconite root, and was originally considered to be effective against 'numbness' due to diabetic peripheral neuropathy. Goshajinkigan is also effective for lumbar canal stenosis, lumbar spondylosis deformans and arteriosclerosis obliterans in the elderly. Similar to the pharmacological effect of Goshajinkigan, it is considered that the analgesic effect is exerted by the suppression of pain-transmitting substance release by $\kappa$-opioid receptor stimulation mediated by dynorphin, an endogenous opioid substance released by ShujiBushi (processed Aconite root) (20). It is also considered that the analgesic effect is exerted through the improvement of peripheral nocireceptor sensitivity, vasodilation and peripheral circulation by the promotion of NO production due to the effects of Takusha (Alisma rhizome) and Sanyaku (Dioscorea rhizome) mediated by bradykinin $\mathrm{B} 2$ receptor and muscarinic acetylcholine receptor (21).

It is expected that Goshajinkigan may become a first-line therapy against the neurotoxicity of anticancer drugs not only by alleviating the subjective symptoms of neuropathy, but also by repairing the nerves. It is essential to delay the progression of neuropathy in current cancer chemotherapy, which should be performed with maintenance of QOL.

\section{References}

1. Kono T, Mishima H, Shimada M, Morita S and Sakamoto J: Preventive effect of goshajinkigan on peripheral neurotoxicity of FOLFOX therapy: a placebo-controlled double-blind randomized phase II study (the GONE Study). Jpn J Clin Oncol 39: 847-849, 2009. 
2. Cella D, Peterman A, Hudgens S, Webster K and Socinski MA Measuring the side effects of taxane therapy in oncology: the functional assesment of cancer therapy-taxane (FACT-taxane). Cancer 15: 822-831, 2003.

3. Takekuma K, Ando F, Niino N and Shimokawa H: Prevalence of hyperesthesia detected by current perception threshold test in subjects with glucose metabolic impairments in a community. Intern Med 41: 1124-1129, 2002.

4. Oishi M, Mochizuki Y, Suzuki Y, Ogawa K, Naganuma T, Nishijo Y and Mizutani T: Current perception threshold and sympathetic skin response in diabetic and alcoholic polyneuropathies. Intern Med 41: 819-822, 2002.

5. Nishimura A, Ogura T, Hase H, Makinodan A,Hojo T, Katsumi Y, Yagi K, Mikami Y and Kubo T: A correlative electrophysiologic study of nerve fiber involvement in carpal tunnel syndrome using current perception thresholds. Clin Neurophysiol 115: 1921-1924, 2004.

6. American Association of Electrodiagnostic Medicine: Technology review: the neurometer ${ }^{\circledR}$ current perception threshold (CPT). Muscle Nerve 22: 523-531, 1999.

7. Noda K, Ikeda M, Kudo R, et al: Phase II study of paclitaxel (BMS-181339) in patients with ovarian cancer by 3-hour intravenous infusion. Gan To Kagaku Ryoho 23: 317-325, 1996.

8. Vasey PA, Jayson GC, Gordon A, et al: Phase III randomized trial of docetaxel-carboplatin versus paclitaxel-carboplatin as first-line chemotherapy for ovarian carcinoma. J Natl Cancer Inst 96: 1682-1691, 2004.

9. Katims JJ, Long DM and Ng LK: Transcutaneous nerve stimulation. Frequency and waveform specificity in humans. App Neurophysiol 49: 86-91, 1986.

10. Apfel SC, Arezzo JC, Lipson L and Kessler JA: Nerve growth factor prevents experimental cisplatin neuropathy. Ann Neurol 31: 76-80, 1992

11. Hayakawa K, Itoh T, Niwa H, Mutoh T and Sobue G: NGF prevention of neurotoxicity induced by cisplatin, vincristine and taxol depends on toxicity of each drug and NGF treatment schedule: in vitro study of adult rat sympathetic ganglion explants. Brain Res 794: 313-319, 1998.
12. Contreras PC, Vaught JL, Gruner JA, Brosnan C, Steffler C, Arezzo JC, Lewis ME, Kessler JA and Apfel SC: Insulin-like growth factor-I prevents development of a vincristine neuropathy in mice. Brain Res 774: 20-26, 1997.

13. Boucher TJ, Okuse K, Bennett DL, Munson JB, Wood JN and McMahon SB: Potent analgesic effects of GDNF in neuropathic pain states. Science 290: 124-127, 2000.

14. Selvaggi $G$ and Belani CP: Carboplatin and paclitaxel in non-small cell lung cancer: the role of amifostine. Semin Oncol 26: 51-60, 1999.

15. Planting AS, Catimel G, de Mulder PH, de Graeff A, Höppener F, Verweij J, Oster W and Vermorken JB: Randomized study of a short course of weekly cisplatin with or without amifostine in advanced head and neck cancer. Ann Oncol 10: 693-700, 1999.

16. Cascinu S, Cordella L, del Ferro E, Fronzoni M and Catalano G: Neuroprotective effect of reduced glutathione on cisplatin-based chemotherapy in advanced gastric cancer: a randomized doubleblind placebo-controlled trial. J Clin Oncol 13: 26-32, 1995.

17. Ziegler D, Reljanovic M, Mehnert H and Gries FA: Alpha-lipoic acid in the treatment of diabetic polyneuropathy in Germany: current evidence from clinical trials. Exp Clin Endocrinol Diabetes 107: 421-430, 1999.

18. Rao RD, Michalak JC, Sloan JA, et al: Efficacy of gabapentin in the management of chemotherapy-induced peripheral neuropathy: a phase 3 randomized, double-blind, placebo-controlled, crossover trial (NO0C3). Cancer 110: 2110-2118, 2007.

19. Eckel F, Schmelz R, Adelsberger H, Erdmann J, Quasthoff S and Lersch C: Prevention of oxaliplatin-induced neuropathy by carbamazepine. A pilot study. Dtsch Med Wochenschr 127: 78-82, 2002.

20. Suzuki Y, Goto K, Ishige A, Komatsu Y and Kamei J: Antinociceptive effect of Gosha-jinki-gan, a Kampo medicine, in streptozotocin-induced diabetic mice. Jpn J Pharmacol 79: 169-175, 1999.

21. Suzuki Y, Goto K, Ishige A, Komatsu Y and Kamei J: Antinociceptive mechanism of Gosha-jinki-gan in streptozotocin-induced diabetic animals: role of nitric oxide in the periphery. Jpn J Pharmacol 79: 387-391, 1999. 